\title{
Improved Communication Efficiency for Distributed Mean Estimation with Side Information
}

\author{
Kai Liang* and Youlong $\mathrm{Wu}^{*}$ \\ * ShanghaiTech University, Shanghai, China \\ $\dagger$ Shanghai Institute of Microsystem and Information Technology, Chinese Academy of Sciences \\ $\ddagger$ University of Chinese Academy of Sciences, Beijing, China \\ \{liangkai,wuyl1\}@shanghaitech.edu.cn
}

\begin{abstract}
In this paper, we consider the distributed mean estimation problem where the server has access to some side information, e.g., its local computed mean estimation or the received information sent by the distributed clients at the previous iterations. We propose a practical and efficient estimator based on an $r$-bit Wynzer-Ziv estimator proposed by Mayekar et al., which requires no probabilistic assumption on the data. Unlike Mayekar's work which only utilizes side information at the server, our scheme jointly exploits the correlation between clients' data and server's side information, and also between data of different clients. We derive an upper bound of the estimation error of the proposed estimator. Based on this upper bound, we provide two algorithms on how to choose input parameters for the estimator. Finally, parameter regions in which our estimator is better than the previous one are characterized.
\end{abstract}

Index Terms-distributed mean estimation, side information, distributed lossy compression

\section{INTRODUCTION}

With the development of modern machine learning technology, more powerful and complex machine learning models can be trained through large-scale distributed training. However, due to the large scale of the model parameters, in each iteration of the distributed optimization, the exchange of information between distributed nodes incurs a huge communication load, causing the problem of communication bottleneck.

We focus on distributed mean estimation, which is a crucial primitive for distributed optimization frameworks. Federated learning [1] is one of such frameworks, in which clients participating in joint training only need to exchange their own gradient information without sharing private data. To alleviate the communication bottleneck, gradient compression $[2]-[8]$ and efficient mean estimator $[9]-[15]$ have been investigated to reduce the communication load. Recently, [16] studied distributed mean estimation with side information at the server, and proposed Wyner-Ziv estimators that require no probabilistic assumption on the clients data.

In parallel, distributed source compression has been widely studied in classical information theory. For example, [17] first studied the setting of lossy source compression with side information in the decoder. Channel coding can obtain practical coding for distributed source coding [18], [19], but the main bottleneck lies in the expensive computational complexity of coding and decoding.

This work is supported by NSFC grant NSF61901267.
In this paper, we study practical schemes for distributed mean estimation with side information at the server. The motivation is based on the fact that the server could store publicly accessible data, and also at each iteration, the server has already received data sent by clients at previous iterations, which can be viewed as side information. Rather than using random coding with joint typicality tools such as in [17], [20], which is impractical to implement, we follow the work in [16] which proposed a Wyner-Ziv estimator based on coset coding. Unfortunately, they only utilized the side information at the server, but failed to exploit correlation between clients' vectors. In fact, in many scenarios such as stochastic gradient descent, data between different clients may have a high correlation since they wish to learn a global model. Inspired by Wyner-Ziv and Slepian-Wolf coding, we propose a practical scheme based on the coset coding and jointly exploit the side information at the server and correlation between clients' data. Note that in our scheme we must address an ambiguity problem not existing in [16] or in the classic WynerZiv coding. In more detail, since each client compresses its data and sends it to the server, the server only observes a lossy version of clients' vectors. This ambiguity cause mismatch information at the clients and server. Using the lossy version of clients' data at the server may even deteriorate the estimation.

We summarize our contributions as follows: 1) We propose a new estimator that improves the estimator in [16] by jointly exploiting the side information at the server and the correlation between clients' data; 2) We derive an upper bound of estimation error of the proposed estimator; 3) We provide two greedy algorithms on how to choose input parameters for the estimator, and characterize the parameter regions in which our estimator has a tighter upper bound than of the previous estimator.

\section{Problem Setting}

Consider the problem of distributed mean estimation with side information, as depicted in Fig. 1. The model consisting of $n$ clients and one server, where each Client $i \in[n] \triangleq$ $\{1, \ldots, n\}$ observes data $x_{i} \in \mathcal{X} \subset \mathbb{R}^{d}$ and the server has access to side information $\boldsymbol{y}=\left(y_{1}, \ldots, y_{n}\right), y_{i} \in \mathcal{Y} \subset \mathbb{R}^{d}$, for some alphabets $\mathcal{X}, \mathcal{Y}$ and positive integer $d \in \mathbb{N}$. The 


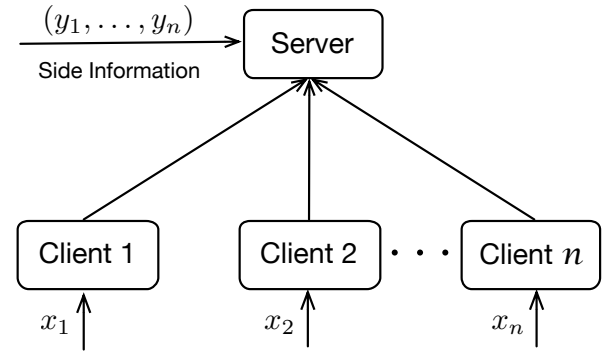

Fig. 1. Distributed mean estimation with side information

server wishes to compute the empirical mean, i.e,

$$
\bar{x} \triangleq \frac{1}{n} \sum_{i=1}^{n} x_{i} .
$$

Note that the side information $\boldsymbol{y}$ could stem from some publicly accessible data or the server's guess of $\boldsymbol{x} \triangleq\left(x_{1}, \ldots, x_{n}\right)$ in the previous iterations.

We focus on non-interactive protocols and study the $r$-bit simultaneous message passing (SMP) protocol similar to that in [16]. The $r$-bit SMP protocol $\pi=\left(\pi_{1}, \ldots, \pi_{n}\right)$ consists of $n$ encoders $\left\{\Psi_{i}\right\}_{i=1}^{n}$ and one decoder $\Phi$, of mapping forms:

$$
\begin{aligned}
& \Psi_{i}: \mathcal{X} \rightarrow\{0,1\}^{r}, \\
& \Phi: \underbrace{\{0,1\}^{r} \times \ldots \times\{0,1\}^{r}}_{n \text { times }} \times \mathcal{Y}^{n} \rightarrow \mathbb{R}^{d} .
\end{aligned}
$$

Each Client $i \in[n]$ uses the encoder $\Psi_{i}$ to encode $x_{i}$ into an $r$-bit message, i.e., $m_{i}=\Psi_{i}\left(x_{i}, U\right)$, where $U$ denotes a shared randomness known by all the server and clients. The Client $i$ then sends the message $m_{i}$ to the server. Assume the message $m_{i}$ can be perfectly received by the server. After receiving all messages $\mathbf{m}^{(n)}=\left(m_{1}, \ldots, m_{n}\right)$, the server uses decoder $\Phi$ to produce $\hat{\bar{x}}$ as

$$
\hat{\bar{x}}=\Phi\left(\mathbf{m}^{(n)}, \boldsymbol{y}, U\right) .
$$

The performance of the $r$-bit SMP protocol using protocol $\pi$ with inputs $\boldsymbol{x}$ and $\boldsymbol{y}$, is evaluated by the mean squared error (MSE), i.e.,

$$
\mathcal{E}_{\pi}(\boldsymbol{x}, \boldsymbol{y}) \triangleq \mathbb{E}\left[\|\hat{\bar{x}}-\bar{x}\|_{2}^{2}\right] .
$$

Instead of using any probabilistic assumption on input data and side information, we use the Euclidean distance between vectors to measure correlation among the data and side information. More specifically, let $x_{i}$ and $y_{i}$ be at most $\Delta_{i}$ and the distance between $x_{i}$ and $x_{j}$ be at most $\Delta_{i j}$, i.e.,

$$
\begin{array}{r}
\left\|x_{i}-y_{i}\right\|_{2} \leq \Delta_{i}, \forall i \in[n], \\
\left\|x_{i}-x_{j}\right\|_{2} \leq \Delta_{i j}, \forall i, j \in[n] .
\end{array}
$$

Since the distance is symmetric with $\Delta_{i j}=\Delta_{j i}$, it's sufficient to only consider $\Delta_{i j}$ with $i<j$. Let

$$
\boldsymbol{\Delta}_{\boldsymbol{s}} \triangleq\left(\Delta_{1}, \cdots, \Delta_{n}\right), \boldsymbol{\Delta}_{c} \triangleq\left(\Delta_{12}, \cdots, \Delta_{(n-1) n}\right) .
$$

We are interested in the performance of protocols when $\boldsymbol{\Delta}_{s}$ and $\boldsymbol{\Delta}_{c}$ are both known to clients and server. Define the optimal $r$-bits protocol with the minimum MSE as $\pi^{*}$, and the corresponding MSE as $\mathcal{E}_{\pi^{*}}(\boldsymbol{x}, \boldsymbol{y})$. Our goal is to find practical and efficient $r$-bits SMP protocols, and derive tighter upper bounds on $\mathcal{E}_{\pi^{*}}$ than the previous results.

\section{Previous Work}

In [16], the authors proposed a SMP protocol based on an $r$-bit Wyner-Ziv quantizer $Q_{\mathrm{Wz}}$. The quantizer $Q_{\mathrm{Wz}}$ contains an encoder mapping $Q_{\mathrm{WZ}}^{\mathrm{e}}$ the same as (2) and a simplified decoder mapping $Q_{\mathrm{WZ}}^{\mathrm{d}}:\{0,1\}^{r} \times \mathcal{Y} \rightarrow \mathbb{R}^{d}$. Each Client $i \in[n]$ first uses the encoder $Q_{\mathrm{WZ}}^{\mathrm{e}}$ to encode $x_{i}$ and then sends the encoded message $m_{i}$ to the server. The server uses the decoder $Q_{\mathrm{WZ}}^{\mathrm{d}}$ to produce estimate $\hat{x}_{i}$ as

$$
\hat{x}_{i}=Q_{\mathrm{WZ}}^{\mathrm{d}}\left(m_{i}, y_{i}\right),
$$

and then computes the sampling means as

$$
\hat{\bar{x}}=\frac{1}{n} \sum_{i=1}^{n} \hat{x}_{i} .
$$

The quantizer $Q_{\mathrm{WZ}}$ achieves the following upper bound on MSE.

Theorem 1 (Upper bound given in [16]). For a fixed $\Delta_{s}$ and $r \leq d$, the optimal $r$-bits protocol $\pi^{*}$ satisfy

$$
\mathcal{E}_{\pi^{*}}(\boldsymbol{x}, \boldsymbol{y}) \leq(79\lceil\log (2+\sqrt{12 \ln n})\rceil+26)\left(\sum_{i=1}^{n} \frac{\Delta_{i}^{2} d}{n^{2} r}\right),
$$

for all $\boldsymbol{x}$ and $\boldsymbol{y}$ satisfying (6).

Now we introduce the quantizer $Q_{\mathrm{WZ}}$, as it is closely related to work. Since all clients use the same quantizer, only the common quantizer is described. We first describe a modulo quantizer $Q_{\mathrm{M}}$ for one-dimension input $x \in \mathbb{R}$ with side information $h \in \mathbb{R}$, and then present a rotated modulo quantizer $Q_{\mathrm{M}, R}(x, h)$ for $d$-dimension data. Finally, the $r$-bit Wyner-Ziv quantizer based on $Q_{\mathrm{M}}$ and $Q_{\mathrm{M}, R}$ is given.

1) Modulo Quantizer $\left(Q_{\mathrm{M}}\right)$ : Given the input $x \in \mathbb{R}$ with side information $h \in \mathbb{R}$, the modulo quantizer $Q_{\mathrm{M}}$ contains parameters including a distance parameter $\Delta^{\prime}$ where $|x-h| \leq$ $\Delta^{\prime}$, a resolution parameter $k \in \mathbb{N}^{+}$and a lattice parameter $\epsilon$.

Denote the encoder and decoder of $Q_{\mathrm{M}}$ as $Q_{\mathrm{M}}^{\mathrm{e}}(x)$ and $Q_{\mathrm{M}}^{\mathrm{d}}\left(Q_{\mathrm{M}}^{\mathrm{e}}(x), h\right)$, respectively. The encoder $Q_{\mathrm{M}}^{\mathrm{e}}(x)$ first computes $\lceil x / \epsilon\rceil$ and $\lfloor x / \epsilon\rfloor$, and then outputs the message $Q_{\mathrm{M}}^{\mathrm{e}}(x)=$ $m$, where

$$
m=\left\{\begin{array}{lll}
(\lceil x / \epsilon\rceil & \bmod k), & \text { w.p. } x / \epsilon-\lfloor x / \epsilon\rfloor \\
(\lfloor x / \epsilon\rfloor \bmod k), & \text { w.p. }\lceil x / \epsilon\rceil-x / \epsilon
\end{array} .\right.
$$

The message $m$ has length of $\log k$ bits, and is sent to the decoder. The decoder $Q_{\mathrm{M}}^{\mathrm{d}}$ produces the estimate $\hat{x}$ by finding a point closest to $h$ in the set $\mathbb{Z}_{m, \epsilon}=\{(z k+m) \cdot \epsilon: z \in \mathbb{Z}\}$.

2) Rotated Modulo Quantizer $\left(Q_{\mathrm{M}, R}\right)$ : Given the input $x \in$ $\mathbb{R}^{d}$ with side information $h \in \mathbb{R}^{d}$ where $\|x-h\|_{2} \leq \Delta$, the input parameters for $Q_{\mathrm{M}, R}$ include a distance parameter $\Delta^{\prime}$, a resolution parameter $k \in \mathbb{N}^{+}$, a lattice parameter $\epsilon$, and a rotation matrix $R$ given by

$$
R=W D / \sqrt{d},
$$


where $W$ is the $d \times d$ Walsh-Hadamard Matrix [21] and $D$ is a diagonal matrix with each diagonal entry generated uniformly from $\{+1,-1\}$ by using a shared randomness. After the rotation, every coordinate $i \in[d]$ of $R(x-h)$, denoted by $R(x-h)(i)$, has zero mean sub-Gaussian with a variance factor of $\Delta^{2} / d$, i.e.,

$$
P\left(|R(x-h)(i)| \geq \Delta^{\prime}\right) \leq 2 e^{-\frac{\Delta^{\prime 2} d}{2 \Delta^{2}}} .
$$

The quantizer $Q_{\mathrm{M}, R}$ first preprocesses $x$ and $h$ by multiplying both $x$ and $h$ with a matrix $R$, and then applies $Q_{\mathrm{M}}$ for each coordinate. Denote the encoder and decoder of $Q_{\mathrm{M}, R}$ as $Q_{\mathrm{M}, R}^{\mathrm{e}}(x)$ and $Q_{\mathrm{M}, R}^{\mathrm{d}}\left(Q_{\mathrm{M}, R}^{\mathrm{e}}(x), h\right)$, respectively.

3) The r-bit Wyner-Ziv Quantizer $\left(Q_{\mathrm{WZ}}\right)$ : Note that in the quantizer $Q_{\mathrm{M}, R}$ the input $x$ is encoded into $d$ binary strings of $\log k$ bits each, leading to a total number of $d \log k$ bits. In the $r$-bit Wyner-Ziv quantizer, the encoder first encodes $x$ using the same encoder as $Q_{\mathrm{M}, R}^{\mathrm{e}}(x)$, and then uses a shared randomness to select a subset $S \subset\{1, \ldots, d\}$ of these strings with $|S|=\lfloor r / \log k\rfloor$, and finally sends them to decoder. The decoder uses the same decoder as $Q_{\mathrm{M}, R}^{\mathrm{d}}$ to decode the entries in $S$. Denote the encoder and decoder of $Q_{\mathrm{WZ}}$ as $Q_{\mathrm{WZ}}^{\mathrm{e}}(x)$ and $Q_{\mathrm{WZ}}^{\mathrm{d}}\left(Q_{\mathrm{WZ}}^{\mathrm{e}}(x), h\right)$, respectively.

\section{New Protocol AND New Upper Bound}

\section{A. New Protocols}

Note that in (8) only $y_{i}$ is used as side information to assist the estimation for $x_{i}$ at the server. In fact, apart from $y_{i}$, the side information $\left\{y_{j}\right\}_{j \neq i}$ and other clients' data $\left\{x_{j}\right\}_{j \neq i}$ could also be correlated to $x_{i}$, and thus can be jointly utilized to reduce the transmission load. The main challenge is that $\left\{x_{j}\right\}_{j \neq i}$ cannot be perfectly known by the server, and thus using the estimate $\left\{\hat{x}_{j}\right\}_{j \neq i}$ as side information for $x_{i}$ may even deteriorate the estimation.

Our protocol is based on a set of $r$-bit new quantizers, denoted by $\left\{Q_{\mathrm{Pro}}^{\mathcal{L}_{\Pi_{i}}}\right\}_{i=1}^{n}$, where $\Pi_{i}$ denotes the $i$-th element of a permutation $\Pi$ of $[n]$, and $\mathcal{L}_{\Pi_{i}}$ is a chain parameter need to be designed and has a form of $\mathcal{L}_{\Pi_{i}}: y_{\Pi_{i_{1}}} \rightarrow x_{\Pi_{i_{1}}} \rightarrow$ $x_{\Pi_{i 2}} \cdots \rightarrow x_{\Pi_{i l}}$, with $x_{\Pi_{i l}}=x_{\Pi_{i}}$ and $l$ being the length of chain.

Given a set of chains $\left\{\mathcal{L}_{\Pi_{i}}: i \in[n]\right\}$, the input data $\left\{x_{i}\right\}_{i=1}^{n}$ are estimated in an order $x_{\Pi_{1}}, \ldots, x_{\Pi_{n}}$. For the input $x_{\Pi_{i}}$, the corresponding quantizer $Q_{\mathrm{Pro}}^{\mathcal{L}_{\Pi_{i}}}$ consists of an encoder the same as $Q_{\mathrm{WZ}}^{\mathrm{e}}\left(x_{\Pi_{i}}\right)$, and a novel decoder $Q_{\mathrm{Pro}}^{\mathrm{d}, \mathcal{L}_{\Pi_{i}}}$ of mapping form

$$
Q_{\text {Pro }}^{\mathrm{d}, \mathcal{L}_{\Pi_{i}}}: \underbrace{\{0,1\}^{r} \times \ldots \times\{0,1\}^{r}}_{i \text { times }} \times \mathcal{Y}^{i} \rightarrow \mathbb{R}^{d},
$$

that is used to decode $x_{\Pi_{i}}$ as

$$
\hat{x}_{Q_{\mathrm{Pro}} \mathcal{L}_{\Pi_{i}}, \Pi_{i}}=Q_{\mathrm{Pro}}^{\mathrm{d}, \mathcal{L}_{\Pi_{i}}}\left(Q_{\mathrm{WZ}}^{\mathrm{e}}\left(x_{\Pi_{1}}\right), \ldots, Q_{\mathrm{WZ}}^{\mathrm{e}}\left(x_{\Pi_{i}}\right), y_{\Pi_{1}}, \ldots, y_{\Pi_{i}}\right) .
$$

Given any quantizer $Q$, denotes its estimate for input $x_{i}$ as $\hat{x}_{Q, i}$. Here $\hat{x}_{Q_{\mathrm{Pro}}, \Pi_{\Pi_{i}}}^{\mathcal{L}_{\Pi_{\pi_{i}}}}$ denotes the estimate for $x_{\Pi_{i}}$ when using the quantizer $Q_{\mathrm{Pro}}$ for the given chain $\mathcal{L}_{\Pi_{i}}$. With a slight abuse of notation, we write $\hat{x}_{Q_{\mathrm{Pro}} \mathcal{L}_{\Pi_{i}}, \Pi_{i}}$ as $\hat{x}_{Q_{\mathrm{Pro}}, \Pi_{i}}$.
In the following, we describe the quantizers $\left\{Q_{\mathrm{Pro}}^{\mathcal{L}_{\Pi_{i}}}\right\}_{i \in[n]}$ in two steps: 1) Given a set of chains $\left\{\mathcal{L}_{\Pi_{i}}\right\}_{i \in[n]}$, how to estimate $\hat{x}_{Q_{\mathrm{Pro}}, \Pi_{i}}$, for $i \in[n]$; 2) How to select proper chains $\left\{\mathcal{L}_{\Pi_{i}}\right\}_{i \in[n]}$ to reduce the MSE.

1) New quantizer for some given chains $\left\{\mathcal{L}_{i}\right\}_{i \in[n]}$ : Without loss of generality, we assume that the estimation order $\Pi$ is an identity permutation, i.e., $\Pi_{i}=i, i \in[n]$. With this assumption, the chain $\mathcal{L}_{\Pi_{i}}$ can be written as

$$
\mathcal{L}_{i}: y_{i_{1}} \rightarrow x_{i_{1}} \rightarrow x_{i_{2}} \cdots \rightarrow x_{i_{l}},
$$

where $x_{i_{l}}=x_{i}, i_{t} \in[i-1]$ for all $t=1, \ldots, l-1$, and the decoder $i$ already has $i-1$ estimates: $\hat{x}_{Q_{\mathrm{Pro}}, 1}, \ldots, \hat{x}_{Q_{\mathrm{Pro}}, i-1}$.

The encoder is same as $Q_{\mathrm{WZ}}^{e}$, i.e., Client $i$ first applies the encoder $Q_{\mathrm{M}, R}^{\mathrm{e}}\left(x_{i}\right)$ to encode $x_{i}$, then uses a shared randomness to select a subset $S \subset\{1, \ldots, d\}$ of these strings with $|S|=\lfloor r / \log k\rfloor$, and finally send them to decoder.

The decoder $Q_{\mathrm{Pro}, i}^{d}$ chooses an element in $\mathcal{M}_{i}$ as the "side" information $h$ for $Q_{\mathrm{M}, R}^{\mathrm{d}}$, where

$$
\mathcal{M}_{i}= \begin{cases}\left\{y_{i}, \hat{x}_{Q_{\mathrm{Pro}}, 1}, \cdots, \hat{x}_{Q_{\mathrm{Pro}}, i-1}\right\}, & \text { if } i>1 \\ \left\{y_{i}\right\}, & \text { if } i=1\end{cases}
$$

We emphasize that here the "side" information $h$ could be the estimate of other client's data, rather than the literal side information $y_{i}$ used in the Wyner-Ziv quantizer $Q_{\mathrm{WZ}}$.

Given the chain $\mathcal{L}_{i}$ in (14), denote $\Delta_{i_{1}}^{\prime}$ and $\Delta_{i_{s} i_{s+1}}^{\prime}$ as weight parameters of subchains $y_{i_{1}} \rightarrow x_{i_{1}}$ and $x_{i_{s}} \rightarrow x_{i_{s+1}}$, respectively. The choices of $\Delta_{i_{1}}^{\prime}$ and $\Delta_{i_{s} i_{s+1}}^{\prime}$ is based on (13), and follows a way similar to that in the quantizer $Q_{\mathrm{M}, R}$. For $t \in[l]$, let $w_{i_{t}} \triangleq \Delta_{i_{1}}^{\prime}+\sum_{s=1}^{t-1} \Delta_{i_{s} i_{s+1}}^{\prime}$.

Given a vector $v \in \mathbb{R}^{d}$ and a subset $S \in[d]$, let $v(S) \triangleq\left(v_{i}\right.$ : $i \in S)$. The decoder estimates $\hat{x}_{Q_{\mathrm{Pro}}, i_{t}}(S)$ as the output values in dimension $S$ of the decoder $Q_{\mathrm{M}, R}^{d}\left(Q_{\mathrm{M}, R}^{\mathrm{e}}\left(x_{i_{t}}\right), \hat{x}_{Q_{\mathrm{Pro}}, i_{t-1}}\right)$ with parameters $\Delta^{\prime}=w_{i_{t}}$ and $h=\hat{x}_{Q_{\mathrm{Pro}}, i_{t-1}}$, and estimate the values of $\hat{x}_{Q_{\mathrm{Pro}}, i_{t}}([n] \backslash S)$ as those values in $y_{i_{t}}$, i.e.,

$$
\begin{aligned}
\hat{x}_{Q_{\mathrm{Pro}}, i_{t}}(S) & =Q_{\mathrm{M}, R}^{d}\left(Q_{\mathrm{M}, R}^{\mathrm{e}}\left(x_{i_{t}}\right), \hat{x}_{Q_{\mathrm{Pro}}, i_{t-1}}\right)(S),(16) \\
\hat{x}_{Q_{\mathrm{Pro}}, i_{t}}([n] \backslash S) & =y_{i_{t}}([n] \backslash S) .
\end{aligned}
$$

By recursively using (16), we can obtain the estimate $\hat{x}_{Q_{\mathrm{Pro}}, i}=\hat{x}_{Q_{\mathrm{Pr} o}, i_{l}}$ for the input $x_{i}$.

In our protocol, the parameters $h$ and $\Delta^{\prime}$ depend the design of chains $\left\{\mathcal{L}_{i}\right\}_{i=1}^{n}, S$ is generated by the shared randomness, and $k, \epsilon$ can be freely assigned. When the length chain $\mathcal{L}_{i}$ is $l=1$, the quantizer $Q_{\text {Pro }}^{\mathcal{L}_{i}}$ reduces to the Wyner-Ziv quantizer $Q_{\mathrm{WZ}}$ if the chosen chain is $\mathcal{L}_{i}:=y_{i} \rightarrow x_{i}$.

2) Selection of Chains: Next we give two algorithms on how to choose chains $\left\{\mathcal{L}_{i}: i \in[n]\right\}$.

Algorithm 1: Given weight parameters $\left\{\Delta_{i}^{\prime}: i \in[n]\right\}$ and $\left\{\Delta_{i j}^{\prime}: i, j \in[n], i<j\right\}$, for Client 1 , we use the chain $\mathcal{L}_{1}=y_{1} \rightarrow x_{1}$ with weight $\Delta_{1}^{\prime}$. For Client $i$, we suppose that the chains $\left\{\mathcal{L}_{j}: j \in[i-1]\right\}$ with weight $w_{j}: j \in[i-1]$ are already known.

Construct $i$ chains $\left\{\mathcal{L}^{i}(j): j \leq i\right\}$ as follows.

$$
\begin{aligned}
& \mathcal{L}^{i}(j)=\mathcal{L}_{j} \rightarrow x_{i}, \text { if } j \in[i-1], \\
& \mathcal{L}^{i}(j)=y_{j} \rightarrow x_{j}, \text { if } j=i .
\end{aligned}
$$


Then, compute the weights $\left\{w^{i}(j): j \in[i]\right\}$ according to

$$
\begin{aligned}
& w^{i}(j)=w_{j}+\Delta_{j i}^{\prime}, \text { if } j \in[i-1], \\
& w^{i}(j)=\Delta_{j}^{\prime}, \text { if } j=i .
\end{aligned}
$$

For each Client $i, \mathcal{L}_{i}$ can be chosen from $i$ candidate chains in $\left\{\mathcal{L}^{i}(j): j \in[i]\right\}$. We choose $\mathcal{L}_{i}=\mathcal{L}^{i}\left(j^{*}\right)$ such that $j^{*} \triangleq \arg \min _{j \in[i]} w^{i}(j)$. We formally describe the method in Algorithm 1]

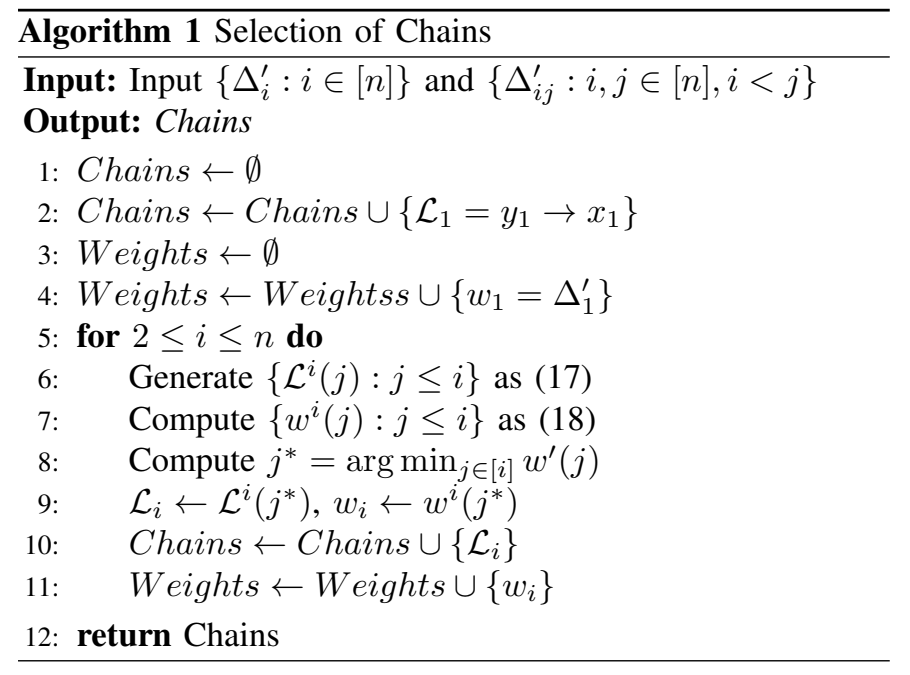

Algorithm 2: Note that Algorithm 1 is simple and fast, but may not find good chains to improve the MSE in (10). Therefore, we are interested in finding good chains and the corresponding region of $\left(\boldsymbol{\Delta}_{\boldsymbol{c}}, \boldsymbol{\Delta}_{\boldsymbol{s}}\right)$ such that the upper bound of MSE is smaller than (10). We illustrate our idea with a special case where the length of each chain is less than 2 .

For Client $i$, consider $y_{t} \rightarrow x_{t} \rightarrow x_{i}$ and $y_{i} \rightarrow x_{i}$ with $t<i$. By Remark 3 (described later in Section IV-B), we select the chain as follows: If $\left(\Delta_{t}, \Delta_{t i}, \Delta_{i}\right) \in \mathcal{R}_{2}$ is in the region $\mathcal{R}_{2}$ defined in 23], then we use the chain $\mathcal{L}_{i}$ as $y_{t} \rightarrow x_{t} \rightarrow x_{i}$, otherwise we use the chain $y_{i} \rightarrow x_{i}$. Now we look for good chains according to $\mathcal{R}_{2}$. Without loss of generality, let $\Delta_{1} \leq$ $\cdots \leq \Delta_{n}$. Starting from Client 1 , firstly, generate a chain of length of 1 for Client 1 , and then traverse the remaining clients to verify whether the corresponding distances are in $\mathcal{R}_{2}$. If the distances are in $\mathcal{R}_{2}$, then construct a chain of length of 2 . For the remaining clients whose chains are empty, renumber them and repeat the above process until every client has a nonempty chain. We formally describe the method in Algorithm 2

\section{B. New Upper Bound of MSE}

Define the following quantities:

$$
\begin{aligned}
& \alpha_{i}(Q) \triangleq \sup _{\boldsymbol{x}, \boldsymbol{y} \text { satisfy }[6]} \mathbb{E}\left[\left\|\hat{x}_{Q, i}-x_{i}\right\|_{2}^{2}\right], \\
& \beta_{i}(Q) \triangleq \sup _{\boldsymbol{x}, \boldsymbol{y} \text { satisfy [6] }}\left\|\mathbb{E}\left[\hat{x}_{Q, i}-x_{i}\right]\right\|_{2}^{2} .
\end{aligned}
$$

Given a chain $\mathcal{L}_{i}$ and a specific parameters assignment, the following lemma gives a recursive inequality about the upper bound of the error when using the quantizer $Q_{\mathrm{Pro}}^{\mathcal{L}_{i}}$.

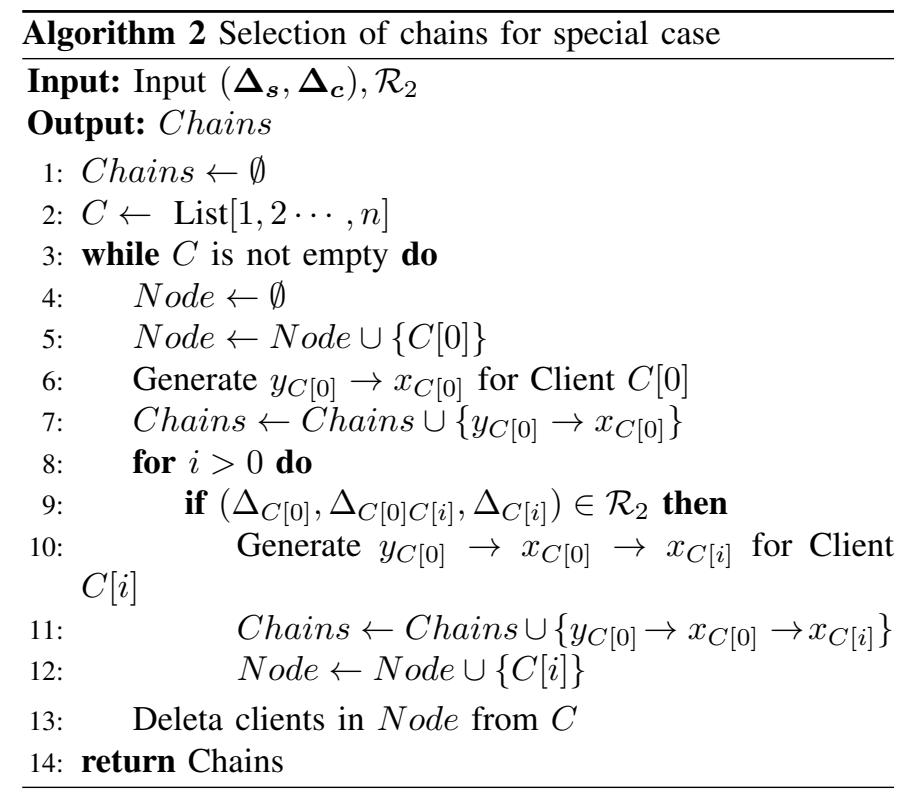

Lemma 1. For a given chain $\mathcal{L}_{i}: y_{i_{1}} \rightarrow x_{i_{1}} \rightarrow x_{i_{2}} \cdots \rightarrow$ $x_{i_{l}}$, when using the quantizer $Q_{\mathrm{Pro}}^{\mathcal{L}_{i}}$ with the following parameters: $k \geq 4, \Delta_{i_{1}}^{\prime}=\sqrt{6\left(\Delta_{i_{1}}^{2} / d\right) \ln \sqrt{n}}, \Delta_{i_{s} i_{s+1}}^{\prime}=$ $\sqrt{6\left(\Delta_{i_{s} i_{s+1}}^{2} / d\right) \ln \sqrt{n}}$, where $s \in[l-1], \mu d=\lfloor r / \log k\rfloor$ and $\epsilon=2 \Delta_{i_{1}}^{\prime} /(k-2)+\sum_{s=1}^{l-1} 2 \Delta_{i_{s} i_{s+1}}^{\prime} /(k-2)$, we have

$$
\begin{aligned}
\alpha_{i_{t}}\left(Q_{\mathrm{Pro}}^{\mathcal{L}_{i_{t}}}\right) \leq & \frac{24 t\left(\Delta_{i_{1}}^{2}+\sum_{s=1}^{t-1} \Delta_{i_{s} i_{(s+1)}}^{2}\right) \ln \sqrt{n}}{\mu(k-2)^{2}} \\
& +c_{t}(n) \frac{\Delta_{i_{1}}^{2}+\sum_{s=1}^{t-1} \Delta_{i_{s} i_{(s+1)}}^{2}+\Delta_{i_{(t-1)} i_{t}}^{2}}{\mu n} \\
& +\frac{3 \alpha_{i_{t-1}}\left(Q_{\mathrm{Pro}}^{\mathcal{L}_{i_{t-1}}}\right)+\Delta_{i_{t}}^{2}}{\mu}, \\
\beta_{i_{t}}\left(Q_{\mathrm{Pro}}^{\mathcal{L}_{i}}\right) \leq & c_{t}(n) \frac{\Delta_{i_{1}}^{2}+\sum_{s=1}^{t-1} \Delta_{i_{s} i_{(s+1)}}^{2}+\Delta_{i_{(t-1)} i_{t}}^{2}}{n} \\
& +3 \alpha_{i_{t-1}}^{2}\left(Q_{\mathrm{Pro}_{i_{t}}}^{\mathcal{L}_{i_{t}}}\right),
\end{aligned}
$$

and

$$
\begin{gathered}
\alpha_{i_{1}}\left(Q_{\mathrm{Pro}}^{\mathcal{L}_{i_{1}}}\right) \leq \frac{24 \Delta_{i_{1}}^{2} \ln \sqrt{n}}{\mu(k-2)^{2}}+154 \frac{\Delta_{i_{1}}^{2}}{\mu n}+\frac{\Delta_{i_{1}}^{2}}{\mu}, \\
\beta_{i_{1}}\left(Q_{\mathrm{PrO}}^{\mathcal{L}_{i_{1}}}\right) \leq 154 \frac{\Delta_{i_{1}}^{2}}{n},
\end{gathered}
$$

for all $t \in[l]$ and $c_{t}(n) \triangleq \max \left\{\frac{576 t^{2}}{e}, 3 n+\frac{36}{e^{2 / 3}}\right\}$.

Proof. See the proof in Appendix A

By properly scaling and choosing an appropriate $k$, we obtain a more concise form in the following corollary, whose proof is given in Appendix B

Corollary 1. If setting $\log k=\lceil\log (2+\sqrt{12 \ln n})\rceil$, we have

$$
\alpha_{i_{l}}\left(Q_{\mathrm{Pro}}^{\mathcal{L}_{i_{l}}}\right) \leq \frac{24 D_{i_{l}}^{i} \ln \sqrt{n}}{\mu(k-2)^{2}}+154 \frac{D_{i_{l}}^{i}}{\mu n}+\frac{\Delta_{i_{l}}^{2}}{\mu},
$$




$$
\beta_{i_{l}}\left(Q_{\mathrm{Pro}}^{\mathcal{L}_{i_{l}}}\right) \leq 154 \frac{D_{i_{l}}^{i}}{n},
$$

and $D_{i_{l}}^{i}$ satisfies that for $l=1, D_{i_{1}}^{i}=\Delta_{1}^{2}$ and for $l>1$,

$$
\begin{aligned}
D_{i_{l}}^{i}= & \max \left\{l\left(\Delta_{i_{1}}^{2}+\sum_{s=1}^{l-1} \Delta_{i_{s} i_{(s+1)}}^{2}\right), \frac{c_{l}(n)}{154}\left(\Delta_{i_{1}}^{2}\right.\right. \\
& \left.\left.+\sum_{s=1}^{l-1} \Delta_{i_{s} i_{(s+1)}}^{2}+\Delta_{i_{(l-1)} i_{l}}^{2}\right)+\frac{3 n D_{i_{l-1}}^{i}}{154}\right\}+3 D_{i_{l_{-1}}}^{i} .
\end{aligned}
$$

Theorem 2. For some fixed $\left(\boldsymbol{\Delta}_{\boldsymbol{s}}, \boldsymbol{\Delta}_{\boldsymbol{c}}\right)$, and $d \geq r \geq 2\lceil\log (2+$ $\sqrt{12 \ln n})\rceil$, and $\mu d=\left\lfloor\frac{r}{\log k}\right\rfloor$, the MSE is upper bounded by

$\mathcal{E}_{\pi^{*}}(\boldsymbol{x}, \boldsymbol{y}) \leq(79 \log k+26) \sum_{i=1}^{n} \frac{d \Delta_{i}^{2}}{n^{2} r}+B \sum_{i=1}^{n} \frac{d\left(D_{i_{l}}^{i}-\Delta_{i}^{2}\right)}{n^{2} r}$,

for all sets of chains $\left\{\mathcal{L}_{i}\right\}_{i=1}^{n}$ and

$$
B= \begin{cases}79 \log k+26, & \text { if } \sum_{i=1}^{n}\left(D_{i_{l}}^{i}-\Delta_{i}^{2}\right) \geq 0 \\ \frac{\log k}{8} . & \text { otherwise }\end{cases}
$$

where $\log k=\lceil\log (2+\sqrt{12 \ln n})\rceil$ and $D_{i_{l}}^{i}$ is given in [21].

Remark 1. We improve the upper bound of MSE in (10) when $\left(\boldsymbol{\Delta}_{c}, \boldsymbol{\Delta}_{s}\right)$ are in the region $\mathcal{R}_{\mathcal{L}}=\left\{\left(\boldsymbol{\Delta}_{c}, \boldsymbol{\Delta}_{s}\right): \sum_{i=1}^{n} D_{i_{l}}^{i}<\right.$ $\left.\sum_{i=1}^{n} \Delta_{i}^{2}\right\}$. In the region $\mathcal{R}_{\mathcal{L}}$ our new upper bound in 22) is $1-\frac{\log k}{8(79 \log k+26)}\left(1-\frac{\sum_{i=1}^{n} D_{i_{l}}^{i}}{\sum_{i=1}^{n} \Delta_{i}^{2}}\right)$ times of that in 10 .

Remark 2. For each permutation $\Pi$ on [n], since each Client $\Pi_{i}$ can choose $\mathcal{L}_{\Pi_{i}}$ from $i$ candidate chains, there are $n$ ! different assignments $\left\{\mathcal{L}_{\Pi_{i}}: i \in[n]\right\}$. Thus, the number of all strategies will not exceed $(n !)^{2}$. Denote the chain corresponding to each strategy as $\mathcal{L}^{i}, i \in\left[(n !)^{2}\right]$. From Theorem 2 and Remark 1, we obtain that when $\left(\boldsymbol{\Delta}_{c}, \boldsymbol{\Delta}_{s}\right) \in \bigcup_{i \in\left[(n !)^{2}\right]} \mathcal{R}_{\mathcal{L}^{i}}$, our upper bound is tighter than that in (10).

Remark 3. If Client $i$ uses the chain $y_{t} \rightarrow x_{t} \rightarrow x_{i}$, by 21), we have $D_{i_{2}}^{i}=\max \left\{2\left(\Delta_{t}^{2}+\Delta_{t i}^{2}\right), \frac{c_{2}(n)}{154}\left(\Delta_{t}^{2}+2 \Delta_{t i}^{2}\right)+\right.$ $\left.\frac{3 n \Delta_{t}^{2}}{154}\right\}+3 \Delta_{t}^{2}$, where $c_{2}(n)=\max \left\{\frac{2304}{e}, 3 n+\frac{36}{e^{2 / 3}}\right\}$. If Client $i$ uses the chain $y_{i} \rightarrow x_{i}$, by 21 , we have $D_{i_{1}}^{i^{2 / 3}}=\Delta_{i}^{2}$. Let

$$
\begin{aligned}
\mathcal{R}_{2} \triangleq & \left\{\left(\Delta_{t}, \Delta_{t i}, \Delta_{i}\right): \max \left\{2\left(\Delta_{t}^{2}+\Delta_{t i}^{2}\right)+3 \Delta_{t}^{2},\right.\right. \\
& \left.\left.c_{2}(n)\left(\Delta_{t}^{2}+2 \Delta_{t i}^{2}\right)+\frac{3 n \Delta_{t}^{2}}{154}+3 \Delta_{t}^{2}\right\}<\Delta_{i}^{2}\right\} .
\end{aligned}
$$

Then, by Theorem 2, if there exists some $i, t \in[n]$ such that $\left(\Delta_{i}, \Delta_{t}, \Delta_{t i}\right) \in \mathcal{R}_{2}$, then our estimator $Q_{\text {pro }}$ improves the Wyner-Ziv estimator $Q_{\mathrm{wz}}$ proposed in [16].

Remark 4. From [23], we observe that to choose a chain whose length is larger than 2, there must exist at least one pair $\left(\Delta_{t}, \Delta_{i}\right)$ such that $5 \Delta_{t}^{2}<\Delta_{i}^{2}$, for some $t, i \in[n]$. Otherwise, our estimator turns to be the Wyner-Ziv estimator in [16]. The condition $5 \Delta_{t}^{2}<\Delta_{i}^{2}$ seems a stringent assumption at the first glance. In fact, since our quantizer is for specific vectors $\boldsymbol{x}$ and $\boldsymbol{y}$, and the Euclidean distance $\left\|x_{i}-y_{i}\right\| \leq \Delta_{i}$ and $\left\|x_{j}-y_{j}\right\| \leq \Delta_{j}$, for $i, j \in[n]$ can vary greatly. Also, one can spend additional bits on better estimating $\Delta_{t}$ and $\Delta_{t i}$ such that they are smaller enough to satisfy [23, and this additional bits cost on estimating $\Delta_{t}$ and $\Delta_{t i}$ can be omitted when $n$ is relatively large.

\section{Proof of Theorem 2}

Now we first introduce a lemma and then derive an upper bound of MSE for any $r$-bit quantizer.

Lemma 2. (see [16]) For $\boldsymbol{x}$ and $\boldsymbol{y}$ satisfying (6), and an $r$ bit quantizer $Q$ using independent randomness for different $i \in[n]$, the estimate $\hat{\bar{x}}$ in (9) and the sample mean $\bar{x}$ satisfies

$$
\mathbb{E}\left[\|\hat{\bar{x}}-\bar{x}\|_{2}^{2}\right] \leq \sum_{i=1}^{n} \frac{\alpha_{i}(Q)}{n^{2}}+\sum_{i=1}^{n} \frac{\beta_{i}(Q)}{n} .
$$

By Lemma 2 , we have

$$
\begin{aligned}
& \mathcal{E}_{\pi^{*}}(\boldsymbol{x}, \boldsymbol{y}) \\
& \leq \sum_{i=1}^{n} \frac{\alpha_{i}\left(Q_{\mathrm{Pro}}^{\mathcal{P}_{i}}\right)}{n^{2}}+\sum_{i=1}^{n} \frac{\beta_{i}\left(Q_{\mathrm{Pro}}^{\mathcal{L}_{i}}\right)}{n} \\
& \stackrel{(a)}{\leq} \frac{r}{\mu d}\left(\frac{24 \ln \sqrt{n}}{(k-2)^{2}}+\frac{154}{n}+154 \mu\right) \sum_{i=1}^{n} \frac{d D_{i_{l}}^{i}}{n^{2} r}+\frac{r}{\mu d} \sum_{i=1}^{n} \frac{d \Delta_{i}^{2}}{n^{2} r} \\
& =\frac{r}{\mu d}\left(\frac{24 \ln \sqrt{n}}{(k-2)^{2}}+\frac{154}{n}+1+154 \mu\right) \sum_{i=1}^{n} \frac{d \Delta_{i}^{2}}{n^{2} r} \\
& +\frac{r}{\mu d}\left(\frac{24 \ln \sqrt{n}}{(k-2)^{2}}+\frac{154}{n}+154 \mu\right) \sum_{i=1}^{n} \frac{d\left(D_{i_{l}}^{i}-\Delta_{i}^{2}\right)}{n^{2} r} \\
& \stackrel{(b)}{\leq}(79\lceil\log (2+\sqrt{12 \ln n})\rceil+26) \sum_{i=1}^{n} \frac{d \Delta_{i}^{2}}{n^{2} r} \\
& +\underbrace{\frac{r}{\mu d}\left(\frac{24 \ln \sqrt{n}}{(k-2)^{2}}+\frac{154}{n}+154 \mu\right)}_{B_{1}} \sum_{i=1}^{n} \frac{d\left(D_{i_{l}}^{i}-\Delta_{i}^{2}\right)}{n^{2} r},
\end{aligned}
$$

where (a) follows by Corollary 1 and $x_{i_{l}}=x_{i}$, and (b) follows by the inequality $\frac{r}{\mu d}\left(\frac{24 \ln \sqrt{n}}{(k-2)^{2}}+\frac{154}{n}+1+154 \mu\right) \leq(79\lceil\log (2+$ $\sqrt{12 \ln n})]+26)$ given in [16]. By this inequality, we can obtain an upper bound of $B_{1}$ as $B_{1} \leq \frac{r}{\mu d}\left(\frac{24 \ln \sqrt{n}}{(k-2)^{2}}+\frac{154}{n}+1+\right.$ $154 \mu) \leq(79\lceil\log (2+\sqrt{12 \ln n})\rceil+26)$, and an lower bound

$$
\begin{aligned}
B_{1} & \geq \frac{r}{\mu d}\left(\frac{24 \ln \sqrt{n}}{(k-2)^{2}}\right) \stackrel{(a)}{\geq} \frac{r}{\mu d}\left(\frac{24 \ln \sqrt{n}}{4(1+\sqrt{12 \ln n})^{2}}\right) \\
& \stackrel{(b)}{\geq} \frac{r}{8 \mu d} \stackrel{(c)}{\geq} \frac{\lceil\log (2+\sqrt{12 \ln n})\rceil}{8},
\end{aligned}
$$

where (a) holds by $\log k \leq \log (2+\sqrt{12 \ln n})+1$, (b) is because $\frac{24 \ln \sqrt{n}}{4(1+\sqrt{12 \ln n})^{2}} \geq \frac{24 \ln \sqrt{2}}{4(1+\sqrt{12 \ln 2})^{2}} \geq 1 / 8$ and (c) holds by $\mu d=\left\lfloor\frac{r}{\log k}\right\rfloor \leq \frac{r}{\log k}$.

\section{CONCLUSION}

In this paper, we studied the distributed mean estimation with limited communication. Inspired by Wyner-Ziv and Slepian-Wolf coding, we proposed new estimator by exploiting the correlation between clients' data. In the future work, we aim to find a more efficient estimator and apply it to more generalized distributed optimization framework. 


\section{REFERENCES}

[1] P. Kairouz, H. B. McMahan, B. Avent, A. Bellet, M. Bennis, A. N Bhagoji, K. Bonawitz, Z. Charles, G. Cormode, R. Cummings et al. "Advances and open problems in federated learning," arXiv preprint arXiv:1912.04977, 2019.

[2] F. Seide, H. Fu, J. Droppo, G. Li, and D. Yu, "1-bit stochastic gradient descent and its application to data-parallel distributed training of speech dnns," in Fifteenth Annual Conference of the International Speech Communication Association, 2014.

[3] H. Wang, S. Sievert, S. Liu, Z. Charles, D. Papailiopoulos, and S. Wright, "Atomo: Communication-efficient learning via atomic sparsification," Advances in Neural Information Processing Systems, vol. 31, pp. 9850-9861, 2018.

[4] D. Alistarh, D. Grubic, J. Li, R. Tomioka, and M. Vojnovic, "Qsgd: Communication-efficient sgd via gradient quantization and encoding," in Advances in Neural Information Processing Systems, 2017, pp. 17091720.

[5] D. Alistarh, T. Hoefler, M. Johansson, N. Konstantinov, S. Khirirat, and C. Renggli, "The convergence of sparsified gradient methods," in Advances in Neural Information Processing Systems, 2018, pp. 59735983.

[6] S. U. Stich, J.-B. Cordonnier, and M. Jaggi, "Sparsified sgd with memory," in Advances in Neural Information Processing Systems, 2018, pp. 4447-4458.

[7] W. Wen, C. Xu, F. Yan, C. Wu, Y. Wang, Y. Chen, and H. Li, "Terngrad: Ternary gradients to reduce communication in distributed deep learning," in Advances in neural information processing systems, 2017, pp. 15091519.

[8] J. Wangni, J. Wang, J. Liu, and T. Zhang, "Gradient sparsification for communication-efficient distributed optimization," Advances in Neural Information Processing Systems, vol. 31, pp. 1299-1309, 2018.

[9] A. T. Suresh, X. Y. Felix, S. Kumar, and H. B. McMahan, "Distributed mean estimation with limited communication," in International Conference on Machine Learning. PMLR, 2017, pp. 3329-3337.

[10] J. Konečnỳ and P. Richtárik, "Randomized distributed mean estimation: Accuracy vs. communication," Frontiers in Applied Mathematics and Statistics, vol. 4, p. 62, 2018.

[11] W.-N. Chen, P. Kairouz, and A. Özgür, "Breaking the communicationprivacy-accuracy trilemma," arXiv preprint arXiv:2007.11707, 2020.

[12] Z. Huang, W. Yilei, K. Yi et al., "Optimal sparsity-sensitive bounds for distributed mean estimation," in Advances in Neural Information Processing Systems, 2019, pp. 6371-6381.

[13] P. Mayekar and H. Tyagi, "Ratq: A universal fixed-length quantizer for stochastic optimization," in International Conference on Artificial Intelligence and Statistics. PMLR, 2020, pp. 1399-1409.

[14] M. Safaryan, E. Shulgin, and P. Richtárik, "Uncertainty principle for communication compression in distributed and federated learning and the search for an optimal compressor," arXiv preprint arXiv:2002.08958, 2020 .

[15] A. Albasyoni, M. Safaryan, L. Condat, and P. Richtárik, "Optimal gradient compression for distributed and federated learning," arXiv preprint arXiv:2010.03246, 2020

[16] P. Mayekar, A. T. Suresh, and H. Tyagi, "Wyner-ziv estimators: Efficient distributed mean estimation with side information," arXiv preprint arXiv:2011.12160, 2020.

[17] A. Wyner and J. Ziv, "The rate-distortion function for source coding with side information at the decoder,' IEEE Transactions on information Theory, vol. 22, no. 1, pp. 1-10, 1976

[18] S. S. Pradhan and K. Ramchandran, "Distributed source coding using syndromes (discus): Design and construction," IEEE transactions on information theory, vol. 49, no. 3, pp. 626-643, 2003.

[19] R. Zamir, S. Shamai, and U. Erez, "Nested linear/lattice codes for structured multiterminal binning," IEEE Transactions on Information Theory, vol. 48, no. 6, pp. 1250-1276, 2002.

[20] S. H. Lim, C. Feng, A. Pastore, B. Nazer, and M. Gastpar, "Towards an algebraic network information theory: Distributed lossy computation of linear functions," in 2019 IEEE International Symposium on Information Theory (ISIT). IEEE, 2019, pp. 1827-1831.

[21] K. J. Horadam, Hadamard matrices and their applications. Princeton university press, 2012.

\section{A. Proof of Lemma 1}

Our quantizer is based on $Q_{\mathrm{M}, R}$, similar to $Q_{\mathrm{WZ}}$. We first introduce the following lemma, whose proof is similar to that in [16]. With a slight abuse of notation, we write $Q_{\mathrm{Pro}}^{\mathcal{L}_{i_{l}}}$ as $Q_{\text {Pro. }}$.

Lemma 3. Fix $\Delta_{i}>0$. Then, for $\mu d \in[d]$, we have

$$
\begin{gathered}
\alpha_{i_{l}}\left(Q_{\text {Pro }}\right) \leq \frac{\alpha_{i_{l}}\left(Q_{\mathrm{M}, R}\right)}{\mu}+\frac{\Delta_{i_{l}}^{2}}{\mu} ; \\
\beta_{i_{l}}\left(Q_{\text {Pro }}\right)=\beta_{i_{l}}\left(Q_{\mathrm{M}, R}\right) .
\end{gathered}
$$

Proof.

$$
\begin{aligned}
& \mathbb{E}\left[\left\|\hat{x}_{Q_{\mathrm{Pro}}, i_{l}}-x_{i_{l}}\right\|_{2}^{2}\right] \\
&=\sum_{j \in[d]} \mathbb{E}\left[\left(\frac{1}{\mu}\left(R \hat{x}_{Q_{\mathrm{M}, R}, i_{l}}(j)-R y_{i_{l}}(j)\right) \mathbb{1}_{\{j \in S\}}\right.\right. \\
&\left.\left.-\left(R x_{i_{l}}(j)-R y_{i_{l}}(j)\right)\right)^{2}\right] \\
&= \sum_{j \in[d]} \mathbb{E}\left[\left(\frac{1}{\mu}\left(R \hat{x}_{Q_{\mathrm{M}, R}, i_{l}}-R x_{i_{l}}(j)\right)\right)^{2} \mathbb{1}_{\{j \in S\}}\right] \\
&+\sum_{j \in[d]} \mathbb{E}\left[\left(\frac{1}{\mu}\left(R x_{i_{l}}(j)-R y_{i_{l}}(j)\right) \mathbb{1}_{\{j \in S\}}\right.\right. \\
&= \frac{1}{\mu} \sum_{j \in[d]} \mathbb{E}\left[\left(R \hat{x}_{Q_{\mathrm{M}, R}, i_{l}}-R x_{i_{l}}(j)\right)^{2}\right] \\
&+\sum_{j \in[d]} \mathbb{E}\left[\left(R x_{i_{l}}(j)-R y_{i_{l}}(j)\right)^{2}\right] \cdot \mathbb{E}\left[\left(\frac{1}{\mu} \mathbb{1}_{\{j \in S\}}-1\right)^{2}\right] \\
&= \frac{1}{\mu} \sum_{j \in[d]} \mathbb{E}\left[\left(R \hat{x}_{Q_{\mathrm{M}, R}, i_{l}}-R x_{i_{l}}(j)\right)^{2}\right] \\
&+\sum_{j \in[d]} \mathbb{E}\left[\left(R x_{i_{l}}(j)-R y_{i_{l}}(j)\right)^{2}\right] \cdot \frac{1-\mu}{\mu} \\
& \leq \frac{\alpha_{i_{l}}\left(Q_{\mathrm{M}, R}\right)}{\mu}+\frac{\Delta_{i_{l}}^{2}}{\mu},
\end{aligned}
$$

where we use the independence of $S$ and $R$ in the third identity and use the fact that $R$ is unitary in the final step.

Since

$$
\begin{aligned}
\left.\| \mathbb{E}\left[\hat{x}_{Q_{\mathrm{Pro}}, i_{l}}\right]-x_{i_{l}}\right) \|_{2}^{2} \\
=\| \sum_{j \in[d]} \mathbb{E}\left[\left(\frac{1}{\mu}\left(R \hat{x}_{Q_{\mathrm{M}, R}, i_{l}}(j)-R y_{i_{l}}(j)\right)\right) \mathbb{1}_{\{j \in S\}}\right. \\
\left.\quad-\left(R x_{i_{l}}(j)-R y_{i_{l}}(j)\right)\right] e_{j} \|_{2}^{2} \\
\left.=\| \mathbb{E}\left[\hat{x}_{Q_{\mathrm{M}, R}, i_{l}}\right]-x_{i_{l}}\right) \|_{2}^{2},
\end{aligned}
$$

where we use the independence of $S$ and $Q_{\mathrm{M}, R}$ in the last identity, we have

$$
\beta_{i_{l}}\left(Q_{\text {Pro }}\right)=\beta_{i_{l}}\left(Q_{\mathrm{M}, R}\right) .
$$


The following lemma is given in [16], which shows that $Q_{\mathrm{M}}$ is unbiased under certain conditions, and the error will not exceed $\epsilon$.

Lemma 4. (see [16]) Consider $Q_{\mathrm{M}}$ described in [II-1] with parameter $\epsilon$ set to satisfy

$$
k \epsilon \geq 2\left(\epsilon+\Delta^{\prime}\right) .
$$

Then, for every $x, h \in \mathbb{R}$ such that $|x-h| \leq \Delta^{\prime}$, the output $Q_{\mathrm{M}}(x)$ satisfies

$$
\begin{gathered}
\mathbb{E}\left[Q_{\mathrm{M}}(x)\right]=x \\
\left|x-Q_{\mathrm{M}}(x)\right|<\epsilon .
\end{gathered}
$$

Recall from Section IV-A that for a chain $y_{i_{1}} \rightarrow x_{i_{1}} \rightarrow$ $x_{i_{2}} \cdots \rightarrow x_{i_{l}}$, the server estimates $x_{i_{l}}$ by using $\hat{x}_{Q_{\mathrm{Pr}, i}, i_{l-1}}^{\mathcal{L}_{i}, i_{l}}$ as $h$ and $\Delta_{i_{1}}^{\prime}+\sum_{s=1}^{l-1} \Delta_{i_{s} i_{(s+1)}}^{\prime}$ as the parameter $\Delta^{\prime}$ in $Q_{\mathrm{M}, R}$. By Lemma 3, we consider the quantizer $Q_{\mathrm{M}, R}$.

Lemma 5. If $R$ given in 12 satisfies that for $j \in[d]$ and $t \in[l],\left|R x_{i_{1}}(j)-R y_{i_{1}}(j)\right| \leq \Delta_{i_{1}}^{\prime},\left|R x_{i_{s}}(j)-R x_{i_{s+1}}(j)\right| \leq$ $\Delta_{i_{s} i_{(s+1)}}^{\prime}, s \in[l-1]$, then, for $k \geq 4$, we have

$$
\left|R x_{i_{t}}(j)-R \hat{x}_{Q_{\mathrm{M}, R}, i_{t-1}}(j)\right| \leq \Delta_{i_{1}}^{\prime}+\sum_{s=1}^{t-1} \Delta_{i_{s} i_{(s+1)}}^{\prime}, t>1,
$$

and if $t=1$, we denote $\hat{x}_{Q_{\mathrm{M}, R}, 0}$ by $y_{i_{1}}$.

Proof. We use induction for $l$. If $l=2$, since $\mid R x_{i_{1}}(j)-$ $R y_{i_{1}}(j) \mid \leq \Delta_{i_{1}}^{\prime}$, by Lemma 4, we have

$$
\left|R x_{i_{1}}(j)-R \hat{x}_{Q_{\mathrm{M}, R}, i_{1}}(j)\right| \leq \frac{2 \Delta_{i_{1}}^{\prime}}{k-2}
$$

where we set $\epsilon=\frac{2 \Delta_{i_{1}}^{\prime}}{k-2}$. Then, for $k \geq 4$

$$
\begin{aligned}
& \left|R x_{i_{2}}(j)-R \hat{x}_{Q_{\mathrm{M}, R}, i_{1}}(j)\right| \\
& \quad \leq\left|R x_{i_{2}}(j)-R x_{i_{1}}(j)+R x_{i_{1}}(j)-R \hat{x}_{Q_{\mathrm{M}, R}, i_{1}}(j)\right| \\
& \quad \leq\left|R x_{i_{2}}(j)-R x_{i_{1}}(j)\right|+\left|R x_{i_{1}}(j)-R \hat{x}_{Q_{\mathrm{M}, R}, i_{1}}(j)\right| \\
& \quad \leq \Delta_{i_{1} i_{2}}^{\prime}+\frac{2 \Delta_{i_{1}}^{\prime}}{k-2} \\
& \quad \leq \Delta_{i_{1} i_{2}}^{\prime}+\Delta_{i_{1}}^{\prime} .
\end{aligned}
$$

Suppose

$$
\left|R x_{i_{t-1}}(j)-R \hat{x}_{Q_{\mathrm{M}, R}, i_{t-2}}(j)\right| \leq \Delta_{i_{1}}^{\prime}+\sum_{s=1}^{t-2} \Delta_{i_{s} i_{(s+1)}}^{\prime} .
$$

By Lemma 4, we have

$$
\left|R x_{i_{t-1}}(j)-R \hat{x}_{Q_{\mathrm{M}, R}, i_{t-1}}(j)\right| \leq \frac{2\left(\Delta_{i_{1}}^{\prime}+\sum_{s=1}^{t-2} \Delta_{i_{s} i_{(s+1)}}^{\prime}\right)}{k-2} .
$$

So, for $t$ and $k \geq 4$, we have

$$
\begin{aligned}
& \left|R x_{i_{t}}(j)-R \hat{x}_{Q_{\mathrm{M}, R}, i_{t-1}}(j)\right| \\
& \quad \leq\left|R x_{i_{t}}(j)-R x_{i_{t-1}}(j)+R x_{i_{t-1}}(j)-R \hat{x}_{Q_{\mathrm{M}, R}, i_{t-1}}(j)\right| \\
& \quad \leq\left|R x_{i_{t}}(j)-R x_{i_{t-1}}(j)\right|+\left|R x_{i_{t-1}}(j)-R \hat{x}_{Q_{\mathrm{M}, R}, i_{t-1}}(j)\right| \\
& \quad \leq \Delta_{i_{(t-1)} i_{t}}^{\prime}+\frac{2\left(\Delta_{i_{1}}^{\prime}+\sum_{s=1}^{t-2} \Delta_{i_{s} i_{(s+1)}}^{\prime}\right)}{k-2} \\
& \quad \leq \Delta_{1}^{\prime}+\sum_{s=1}^{t-1} \Delta_{i_{s} i_{(s+1)}}^{\prime} .
\end{aligned}
$$

For convenience, let $\mathcal{A}_{1}$ denote event $\left\{R: \mid R x_{i_{1}}(j)-\right.$ $\left.R y_{i_{1}}(j) \mid \leq \Delta_{i_{1}}^{\prime}\right\}$ and $\mathcal{A}_{s}$ denote event $\left\{R: \mid R x_{i_{s-1}}(j)-\right.$ $\left.R x_{i_{s}}(j) \mid \leq \Delta_{i_{(s-1)} i_{s}}^{\prime}\right\}$. So, by Lemma 5 , we have that if $R \in \bigcap_{s \in[t]} \mathcal{A}_{s}$, then

$$
\left|R x_{i_{t}}(j)-R \hat{x}_{Q_{\mathrm{M}, R}, i_{t-1}}(j)\right| \leq \Delta_{i_{1}}^{\prime}+\sum_{s=1}^{t-1} \Delta_{i_{s} i_{(s+1)}}^{\prime} .
$$

Thus,

$$
\begin{aligned}
\left|R x_{i_{t}}(j)-R \hat{x}_{Q_{\mathrm{M}, R}, i_{t}}(j)\right| & \leq \epsilon_{i_{1}}+\sum_{s=1}^{t-1} \epsilon_{i_{s} i_{(s+1)}} \\
& =\frac{2\left(\Delta_{i_{1}}^{\prime}+\sum_{s=1}^{t-1} \Delta_{i_{s} i_{(s+1)}}^{\prime}\right)}{k-2} .
\end{aligned}
$$

where we set $\epsilon_{i_{1}}=\frac{2 \Delta_{i_{1}}^{\prime}}{k-2}$ and $\epsilon_{i_{s} i_{(s+1)}}=\frac{2 \Delta_{i_{s} i_{(s+1)}}^{\prime}}{k-2}$.

For the random matrix $R$ given in 112 , for every $z \in \mathbb{R}^{d}$, the random variables $R \boldsymbol{z}(i), i \in[d]$, are sub-Gaussian with variance parameter $\|\boldsymbol{z}\|_{2}^{2} / d$. Furthermore, we need the following bound.

Lemma 6 (see [16]). For a sub-Gaussian random $Z$ with variance factor $\sigma^{2}$ and every $t \geq 0$, we have

$$
\mathbb{E}\left[Z^{2} \mathbb{1}_{\{|Z|>t\}}\right] \leq 2\left(2 \sigma^{2}+t^{2}\right) e^{-t^{2} / 2 \sigma^{2}} .
$$

We now handle the $\alpha_{i_{t}}\left(Q_{\mathrm{M}, R}\right)$ and $\beta_{i_{t}}\left(Q_{\mathrm{M}, R}\right)$ separately below.

Firstly, we consider $t>1$. Since $R$ is a unitary transform, we have

$$
\begin{aligned}
& \mathbb{E}\left[\left\|\hat{x}_{Q_{\mathrm{M}, R}, i_{t}}-x_{i_{t}}\right\|_{2}^{2}\right] \\
& \quad=\mathbb{E}\left[\left\|R \hat{x}_{Q_{\mathrm{M}, R}, i_{t}}-R x_{i_{t}}\right\|_{2}^{2}\right] \\
& =\sum_{j=1}^{d} \mathbb{E}\left[\left(R \hat{x}_{Q_{\mathrm{M}, R}, i_{t}}(j)-R x_{i_{t}}(j)\right)^{2}\right] \\
& =\sum_{j=1}^{d} \mathbb{E}\left[\left(R \hat{x}_{Q_{\mathrm{M}, R}, i_{t}}(j)-R x_{i_{t}}(j)\right)^{2} \mathbb{1}_{\left.\bigcap_{s \in[t]} \mathcal{A}_{s}\right]}\right. \\
& \quad+\sum_{j=1}^{d} \mathbb{E}\left[\left(R \hat{x}_{Q_{\mathrm{M}, R}, i_{t}}(j)-R x_{i_{t}}(j)\right)^{2} \mathbb{1}_{\left.\bigcup_{s \in[t]} \mathcal{A}_{s}^{c}\right] .}\right.
\end{aligned}
$$

We consider the first term. By [27], we have

$$
\begin{aligned}
& \sum_{j=1}^{d} \mathbb{E} {\left[\left(R \hat{x}_{Q_{\mathrm{M}, R}, i_{t}}(j)-R x_{i_{t}}(j)\right)^{2} \mathbb{1}_{\cap_{s \in[t]} \mathcal{A}_{s}}\right] } \\
& \leq d\left(\epsilon_{i_{1}}+\sum_{s=1}^{t-1} \epsilon_{i_{s} i_{(s+1)}}\right)^{2} \\
& \quad \leq d t\left(\epsilon_{i_{1}}^{2}+\sum_{s=1}^{t-1} \epsilon_{i_{s} i_{(s+1)}}^{2}\right),
\end{aligned}
$$

where in the final steps we use the fact that $\left(a_{1}+\cdots+a_{n}\right)^{2} \leq$ $n\left(a_{1}^{2}+\cdots+a_{n}^{2}\right)$. 
For the second term on (28), we get

$$
\begin{aligned}
& \sum_{j=1}^{d} \mathbb{E}\left[\left(R \hat{x}_{Q_{\mathrm{M}, R}, i_{t}}(j)-R x_{i_{t}}(j)\right)^{2} \mathbb{1}_{\left.\cup_{s \in[t]} \mathcal{A}_{s}^{c}\right]}\right. \\
& \leq 3 \sum_{j=1}^{d}\left[\mathbb { E } \left[\left(R \hat{x}_{Q_{\mathrm{M}, R}, i_{t}}(j)-R \hat{x}_{Q_{\mathrm{M}, R}, i_{t-1}}(j)\right)^{2} \mathbb{1}_{\left.\bigcup_{s \in[t]} \mathcal{A}_{s}^{c}\right]}\right.\right. \\
& +\mathbb{E}\left[\left(R \hat{x}_{Q_{\mathrm{M}, R}, i_{t-1}}(j)-R x_{i_{t-1}}(j)\right)^{2} \mathbb{1}_{\bigcup_{s \in[t]} \mathcal{A}_{s}^{c}}\right] \\
& \left.+\mathbb{E}\left[\left(R x_{i_{t-1}}(j)-R x_{i_{t}}(j)\right)^{2} \mathbb{1}_{\bigcup_{s \in[t]}} \mathcal{A}_{s}^{c}\right]\right] \\
& \leq 3 k^{2}\left(\epsilon_{i_{1}}+\sum_{s=1}^{t-1} \epsilon_{i_{s} i_{(s+1)}}\right)^{2} \sum_{j=1}^{d} P\left(\bigcup_{s \in[t]} \mathcal{A}_{s}^{c}\right) \\
& +3 \sum_{j=1}^{d}\left[\mathbb{E}\left[\left(R \hat{x}_{Q_{\mathrm{M}, R}, i_{t-1}}(j)-R x_{i_{t-1}}(j)\right)^{2} \mathbb{1}_{\left.\cup_{s \in[t]} \mathcal{A}_{s}^{c}\right]}\right]\right. \\
& \left.+\mathbb{E}\left[\left(R x_{i_{t-1}}(j)-R x_{i_{t}}(j)\right)^{2} \mathbb{1}_{\bigcup_{s \in[t]}} \mathcal{A}_{s}^{c}\right]\right] \\
& \leq 3 k^{2}\left(\epsilon_{i_{1}}+\sum_{s=1}^{t-1} \epsilon_{i_{s} i_{(s+1)}}\right)^{2} \sum_{j=1}^{d} P\left(\bigcup_{s \in[t]} \mathcal{A}_{s}^{c}\right) \\
& +3 \sum_{j=1}^{d} \mathbb{E}\left[\left(R x_{i_{t-1}}(j)-R x_{i_{t}}(j)\right)^{2} \mathbb{1}_{\bigcup_{s \in[t]} \mathcal{A}_{s}^{c}}\right]+3 \alpha_{i_{t-1}}\left(Q_{\mathrm{M}, R}\right) \\
& \leq 3 k^{2}\left(\epsilon_{i_{1}}+\sum_{s=1}^{t-1} \epsilon_{i_{s} i_{(s+1)}}\right)^{2} \sum_{j=1}^{d} P\left(\bigcup_{s \in[t]} \mathcal{A}_{s}^{c}\right) \\
& +3 \sum_{j=1}^{d} \mathbb{E}\left[\left(R x_{i_{t-1}}(j)-R x_{i_{t}}(j)\right)^{2} \mathbb{1}_{\left.\bigcup_{s \in[t-1]} \mathcal{A}_{s}^{c}\right]}\right. \\
& +3 \sum_{j=1}^{d} \mathbb{E}\left[\left(R x_{i_{t-1}}(j)-R x_{i_{t}}(j)\right)^{2} \mathbb{1}_{\mathcal{A}_{l}^{c}}\right]+3 \alpha_{i_{t-1}}\left(Q_{\mathrm{M}, R}\right) \\
& \leq 3 k^{2}\left(\epsilon_{i_{1}}+\sum_{s=1}^{t-1} \epsilon_{i_{s} i_{(s+1)}}\right)^{2} \sum_{j=1}^{d} P\left(\bigcup_{s \in[t]} \mathcal{A}_{s}^{c}\right) \\
& +3 \Delta_{i_{(t-1)} i_{t}}^{2}+3 \sum_{j=1}^{d} \mathbb{E}\left[\left(R x_{i_{t-1}}(j)-R x_{i_{t}}(j)\right)^{2} \mathbb{1}_{\mathcal{A}_{t}^{c}}\right] \\
& +3 \alpha_{i_{t-1}}\left(Q_{\mathrm{M}, R}\right) \text {. }
\end{aligned}
$$

Since

$$
\begin{aligned}
& P\left(\bigcup_{s \in[t]} \mathcal{A}_{s}^{c}\right) \\
& \leq \sum_{s=1}^{t} P\left(\mathcal{A}_{s}^{c}\right) \\
& \leq 2 e^{-d \Delta_{i_{1}}^{\prime 2} / 2 \Delta_{i_{1}}^{2}}+2 \sum_{s=2}^{t} e^{-d \Delta_{i_{(s-1)} i_{s}}^{\prime 2} / 2 \Delta_{i_{(s-1)} i_{s}}^{2}} \\
& =2 t(\sqrt{n})^{-3},
\end{aligned}
$$

where in the final step we use $\Delta_{i_{1}}^{\prime}=\sqrt{6\left(\Delta_{i_{1}}^{2} / d\right) \ln \sqrt{n}}$ and

$$
\Delta_{i_{(s-1)} i_{s}}^{\prime}=\sqrt{6\left(\Delta_{i_{(s-1)} i_{s}}^{2} / d\right) \ln \sqrt{n}} \text {, we get }
$$

$$
\begin{aligned}
\sum_{j=1}^{d} \mathbb{E}[ & \left(R \hat{x}_{Q_{\mathrm{M}, R}, i_{t}}(j)-R x_{i_{t}}(j)\right)^{2} \mathbb{1}_{\left.\cup_{s \in[t]} \mathcal{A}_{s}^{c}\right]} \\
\leq & 6 t k^{2}\left(\epsilon_{i_{1}}+\sum_{s=1}^{t-1} \epsilon_{i_{s} i_{(s+1)}}\right)^{2}(\sqrt{n})^{-3} \\
& +3 \Delta_{i_{(t-1)} i_{t}}^{2}+3 \sum_{j=1}^{d} \mathbb{E}\left[\left(R x_{i_{t-1}}(j)-R x_{i_{t}}(j)\right)^{2} \mathbb{1}_{\mathcal{A}_{t}^{c}}\right] \\
& +3 \alpha_{i_{t-1}}\left(Q_{\mathrm{M}, R}\right) \\
(a) & 6 t k^{2}\left(\epsilon_{i_{1}}+\sum_{s=1}^{t-1} \epsilon_{i_{s} i_{(s+1)}}\right)^{2}(\sqrt{n})^{-3} \\
& +3 \Delta_{i_{(t-1)} i_{t}}^{2}+12 \Delta_{i_{(t-1)} i_{t}}^{2}(1+3 \ln \sqrt{n})(\sqrt{n})^{-3} \\
& +3 \alpha_{i_{t-1}}\left(Q_{\mathrm{M}, R}\right) \\
\leq & 6 t^{2} k^{2}\left(\epsilon_{i_{1}}^{2}+\sum_{s=1}^{t-1} \epsilon_{i_{s} i_{(s+1)}}^{2}\right)(\sqrt{n})^{-3} \\
& +3 \Delta_{i_{(t-1)} i_{t}}^{2}+12 \Delta_{i_{(t-1)} i_{t}}^{2}(1+3 \ln \sqrt{n})(\sqrt{n})^{-3} \\
& +3 \alpha_{i_{t-1}}\left(Q_{\mathrm{M}, R}\right),
\end{aligned}
$$

where in (a) we use Lemma 6 .

Combining 29] and (32), we have

$$
\begin{aligned}
\mathbb{E} & {\left[\left\|\hat{x}_{Q_{\mathrm{M}, R}, i_{t}}-x_{i_{t}}\right\|_{2}^{2}\right] } \\
\leq & d t\left(\epsilon_{i_{1}}^{2}+\sum_{s=1}^{t-1} \epsilon_{i_{s} i_{(s+1)}}^{2}\right)+6 t^{2} k^{2}\left(\epsilon_{i_{1}}^{2}+\sum_{s=1}^{t-1} \epsilon_{i_{s} i_{(s+1)}}^{2}\right)(\sqrt{n})^{-3} \\
& +3 \Delta_{i_{(t-1)} i_{t}}^{2}+12 \Delta_{i_{(t-1)} i_{t}}^{2}(1+3 \ln \sqrt{n})(\sqrt{n})^{-3}+3 \alpha_{i_{t-1}}\left(Q_{\mathrm{M}, R}\right) \\
\stackrel{(a)}{=} & \frac{24 t A_{i_{t}} \ln \sqrt{n}}{(k-2)^{2}}+\frac{144 t^{2} k^{2} A_{i_{t}} \ln \sqrt{n}}{(k-2)^{2} n \sqrt{n}}+3 \Delta_{i_{(t-1)} i_{t}}^{2} \\
& +12 \Delta_{i_{(t-1)} i_{t}}^{2}(1+3 \ln \sqrt{n})(\sqrt{n})^{-3}+3 \alpha_{i_{t-1}}\left(Q_{\mathrm{M}, R}\right) \\
(\text { b) } & \frac{24 t A_{i_{t}} \ln \sqrt{n}}{(k-2)^{2}}+\frac{576 t^{2} A_{i_{t}}}{e n}+3 \Delta_{i_{(t-1)} i_{t}}^{2}+\frac{36 \Delta_{i_{(t-1)} i_{t}}^{2}}{e^{2 / 3} n} \\
& +3 \alpha_{i_{t-1}}\left(Q_{\mathrm{M}, R}\right) \\
\leq & \frac{24 l A_{i_{t}} \ln \sqrt{n}}{(k-2)^{2}}+c_{t}(n) \frac{A_{i_{t}}+\Delta_{i_{(t-1)} i_{t}}^{2}}{n}+3 \alpha_{i_{t-1}}\left(Q_{\mathrm{M}, R}\right),
\end{aligned}
$$

where in (a) we use $\epsilon_{1}=\frac{2 \Delta_{1}^{\prime}}{k-2}$ and $\epsilon_{(s-1) s}=\frac{2 \Delta_{(s-1) s}^{\prime}}{k-2}$ and in (b) we use $(1+3 \ln \sqrt{n}) / \sqrt{n} \leq 3 / e^{2 / 3}$ and $(\ln \sqrt{n}) / \sqrt{n} \leq$ $1 / e$ and $A_{i_{t}}=\Delta_{i_{1}}^{2}+\sum_{s=1}^{t-1} \Delta_{i_{s} i_{(s+1)}}^{2}$. 
Now, we consider $\beta_{i_{t}}\left(Q_{\mathrm{M}, R}\right)$. For $\beta_{i_{t}}\left(Q_{\mathrm{M}, R}\right)$, we have

$$
\begin{aligned}
& \left\|\mathbb{E}\left[\hat{x}_{Q_{\mathrm{M}, R}, i_{t}}\right]-x_{i_{t}}\right\|_{2}^{2} \\
& \quad=\left\|\mathbb{E}\left[R \hat{x}_{Q_{\mathrm{M}, R}, i_{t}}\right]-R x_{i_{t}}\right\|_{2}^{2} \\
& \quad \stackrel{(a)}{\leq} \sum_{j=1}^{d} \mathbb{E}\left[\left(R \hat{x}_{Q_{\mathrm{M}, R}, i_{t}}(j)-R x_{i_{t}}(j)\right) \mathbb{1}_{\cup_{s \in[t]} \mathcal{A}_{s}^{c}}\right]^{2}, \\
& \quad \leq \sum_{j=1}^{d} \mathbb{E}\left[\left(R \hat{x}_{Q_{\mathrm{M}, R}, i_{t}}(j)-R x_{i_{t}}(j)\right)^{2} \mathbb{1}_{\cup_{s \in[t]}} \mathcal{A}_{s}^{c}\right] \\
& \quad \leq c_{t}(n) \frac{A_{i_{t}}+\Delta_{i_{(t-1)} i_{t}}^{2}}{n}+3 \alpha_{i_{t-1}}\left(Q_{\mathrm{M}, R}\right),
\end{aligned}
$$

where (a) holds by the fact that if $R \in \bigcap_{s \in[t]} \mathcal{A}_{s}$, then $\hat{x}_{Q_{\mathrm{M}, R}, i_{t}}$ is an unbiased estimte of $x_{i_{t}}$ by Lemma 4

For $t=1$, following a similar method above we have

$$
\begin{aligned}
\mathbb{E} & {\left[\left\|\hat{x}_{Q_{\mathrm{M}, R}, i_{1}}-x_{i_{1}}\right\|_{2}^{2}\right] } \\
& \leq d \epsilon_{i_{1}}^{2}+4 d k^{2} \epsilon_{i_{1}}^{2}(\sqrt{n})^{-3}+4\left(2 \Delta_{i_{1}}^{2}+d \Delta_{i_{1}}^{\prime 2}\right)(\sqrt{n})^{-3} \\
& \leq \frac{24 \Delta_{i_{1}}^{2} \ln \sqrt{n}}{(k-2)^{2}}+\left(\frac{96}{e}\left(\frac{k}{k-2}\right)^{2}+\frac{24}{e^{2 / 3}}\right) \frac{\Delta_{i_{1}}^{2}}{n} \\
& \leq \frac{24 \Delta_{i_{1}}^{2} \ln \sqrt{n}}{(k-2)^{2}}+154 \frac{\Delta_{i_{1}}^{2}}{n} .
\end{aligned}
$$

Similarly, for $\beta_{i_{1}}\left(Q_{\mathrm{M}, R}\right)$ we have

$$
\left\|\mathbb{E}\left[\hat{x}_{Q_{\mathrm{M}, R}, i_{1}}\right]-x_{1} i_{1}\right\|_{2}^{2} \leq 154 \frac{\Delta_{i_{1}}^{2}}{n} .
$$

We complete the proof of the lemma according Lemma 3

\section{B. Proof of Corollary 1}

We first consider $\alpha_{i_{l}}\left(Q_{\mathrm{M}, R}\right)$ and $\beta_{i_{l}}\left(Q_{\mathrm{M}, R}\right)$. Then, we prove

$$
\begin{gathered}
\alpha_{i_{l}}\left(Q_{\mathrm{M}, R}\right) \leq \frac{24 D_{i_{l}}^{i} \ln \sqrt{n}}{(k-2)^{2}}+154 \frac{D_{i_{l}}^{i}}{n}, \\
\beta_{i_{l}}\left(Q_{\mathrm{M}, R}\right) \leq 154 \frac{D_{i_{l}}^{i}}{n} .
\end{gathered}
$$

We use induction for $l$. If $l=1$, it is obvious.

Now, we suppose

$$
\begin{gathered}
\alpha_{i_{l-1}}\left(Q_{\mathrm{M}, R}\right) \leq \frac{24 D_{i_{l-1}}^{i} \ln \sqrt{n}}{(k-2)^{2}}+154 \frac{D_{i_{l-1}}^{2}}{n}, \\
\beta_{i_{l-1}}\left(Q_{\mathrm{M}, R}\right) \leq 154 \frac{D_{i_{l-1}}^{i}}{n} .
\end{gathered}
$$

By 33 and (34), we have

$$
\begin{aligned}
& \alpha_{i_{l}}\left(Q_{\mathrm{M}, R}\right) \\
& \leq \frac{24 l A_{i_{l}} \ln \sqrt{n}}{(k-2)^{2}}+c_{i_{l}}(n) \frac{A_{i_{l}}+\Delta_{i_{(l-1)} i_{l}}^{2}}{n}+3 \alpha_{i_{l-1}}\left(Q_{\mathrm{M}, R}\right) \\
& \leq \frac{24\left(l A_{i_{l}}+3 D_{i_{l-1}^{i}}\right) \ln \sqrt{n}}{(k-2)^{2}}+\left(c_{i_{l}}(n) \frac{A_{i_{l}}+\Delta_{i_{(l-1)} i_{l}}^{2}}{154}+3 D_{i_{l-1}^{i}}\right) \cdot \frac{154}{n}
\end{aligned}
$$

and

$$
\begin{aligned}
& \beta_{i_{l}}\left(Q_{\mathrm{M}, R}\right) \\
& \leq c_{i_{l}}(n) \frac{A_{i_{l}}+\Delta_{i_{(l-1)} i_{l}}^{2}}{n}+3 \alpha_{i_{l-1}}\left(Q_{\mathrm{M}, R}\right) \\
& \leq \frac{3 D_{i_{l-1}}^{i} 24 \ln \sqrt{n}}{(k-2)^{2}}+\left(c_{i_{l}}(n) \frac{A_{i_{l}}+\Delta_{i_{(l-1)} i_{l}}^{2}}{154}+3 D_{i_{l-1}^{i}}\right) \cdot \frac{154}{n} \\
& \leq\left(\frac{3 n D_{i_{l-1}}^{i} 24 \ln \sqrt{n}}{154(k-2)^{2}}+c_{i_{l}}(n) \frac{A_{i_{l}}+\Delta_{i_{(l-1)} i_{l}}^{2}}{154}+3 D_{i_{l-1}^{i}}\right) \cdot \frac{154}{n} \\
& \leq\left(\frac{3 n D_{i_{l-1}}^{i}}{154}+c_{i_{l}}(n) \frac{A_{i_{l}}+\Delta_{i_{(l-1)} i_{l}}^{2}}{154}+3 D_{i_{l-1}^{i}}\right) \cdot \frac{154}{n},
\end{aligned}
$$

where in the last step, we use the fact $\frac{24 \ln \sqrt{n}}{(k-2)^{2}} \leq 1$ since $\log k=\lceil\log (2+\sqrt{12 \ln n})\rceil$.

Since

$$
\begin{aligned}
D_{i_{l}}^{i}= & \max \left\{l\left(\Delta_{i_{1}}^{2}+\sum_{s=1}^{l-1} \Delta_{i_{s} i_{(s+1)}}^{2}\right), \frac{c_{l}(n)}{154}\left(\Delta_{i_{1}}^{2}\right.\right. \\
& \left.\left.+\sum_{s=1}^{l-1} \Delta_{i_{s} i_{(s+1)}}^{2}+\Delta_{i_{(l-1)} i_{l}}^{2}\right)+\frac{3 n D_{i_{l-1}}^{i}}{154}\right\}+3 D_{i_{l-1}}^{i},
\end{aligned}
$$

and and Lemma 3 , we complete the proof. 